\title{
“Spontaneous Spinal Epidural Hematoma: A Rare Presentation"
}

\author{
Author: Dr. Bharat Veer Manchanda ${ }^{1}$,Dr. Umesh Verma ${ }^{2}$,Dr. Saloni Mehra ${ }^{3}$, \\ Dr. Girish Dubey ${ }^{4}$,Dr. Shirobhi Sharma ${ }^{5}$,Dr. Arun Khatri ${ }^{6}$ \\ ${ }^{1}$ Assi. Professor In Dept Of Medicine, Subharti Medical College, Meerut \\ ${ }^{2,4,5,6}$ Post Graduate In Dept Of Medicine ,Subharti Medical College, Meerut \\ ${ }^{3}$ Post Graduate In Dept Of Surgery, Subharti Medical College, Meerut
}

\begin{abstract}
Spontaneous spinal epidural hematoma (SSHE) is a rare entity can have several reasons. Its prevalence in population is 0.1 per 100,000 with the male to female ratio of 1/4:1. For the first time Jackson in 1869 reported a case of SSHE and after that, it was declared as several hundred cases in literatures. Here, a case of SSHE was reported in a 22-year-old male referred to emergency department following severe low back pain
\end{abstract}

\section{Introduction}

Spinal epidural hematomas which usually occur in the cervical and thoracic regions are comparatively rare pathologies causing compression of the spinal cord. Clinically it can present through a wide neurological deficit ranging from simple cervical radiculopathy to complete quadriplegia depending on the severity and rapidity of compression. They have been reported in association with blood dyscrasias, coagulopathies, anticoagulant treatment, infection, tumor, pregnancy and vascular malformations but the vast majority doesn't have a definite cause. The spinal epidural hematoma of cervical spine is usually spontaneous and of acute onsetwhile hematomas occurring at lower levels of the spine have a more subacute or chronic course. Moreover, in the latter, the cause of the bleeding is more likely to be definedPatients with this condition usually often require emergency attention because the hematomas can trigger acute neurological deficits. However, there are some cases in which patients demonstrate only mild neurological deficits or non progressive deficits, and can be treated conservatively particularly, some spinal epidural hematomas cases with rapid clinical and radiological resolution have been reported .

\section{Case Report}

A 22 year old male presented with chief complain of paraplegia. Initially episode of lower backache followed by tingling sensation and numbness followed by inability to move both lower limbs. Brain computed tomography was performed, and no abnormality was found, the MRI whole spine was performed and it was suggestive of T1hyperintense lesion in posterior epidural space at D6 level with single change in spinal as described likely epidural hematoma with secondary compressive myelopathy,

He was managed conservatively

\section{Discussion}

Patients with back pain are the common referrals to the ED and consist of $2.3 \%$ of all physician visits. In addition, $84 \%$ of adults experience the back pain in their life. Some important elements in the history and physical examination can differentiate serious reasons of the back pain. The important point in this case was the presence of severe pain resistant to the treatment, which was not mentioned as red flags. SSHE is an idiopathic aggregation of blood in epidural space which can be as acute, chronic, spontaneously, post traumatic, or iatrogenic. SSEH concludes less than one percent of space occupied lesions; its pathogenesis is still unknown but believed that bleeding has a venous origin because the lack of valves in the epidural network disposes the intrinsic change in pressure. Albeit in terms of recent theories, it is supposed that arterial bleeding causes to traction and mechanic disruptions on nerve roots and in some studies, it was attributed to the spontaneous rupture of an arteriovenous malformation. Miyagi et al. declared that because the pressure of intravenous network is lesser than epidural space, arterial bleeding is more possible for that. Totally, epidural hematoma is along with a series of underlying diseases and predisposing factors such as organic vascular disease, hemodialysis, coagulation disorders, hemophilia B, thrombolysis for myocardial infarct, factor IX deficiency, long term aspirin using, and vascular malformations as well as vertebral hemangiomas; liver disease, alcoholism, and thrombocytopenia . The patient usually refers with a sudden sharp and radicular low back pain. The pain maybe exacerbated by percussion on the spine or with maneuvers that increase the intraspinal pressure like coughing and sneezing. The sensory and motor findings of the patient depend on the lesion site and 
hematoma size which can include weakness, paresis, bladder dysfunction, and sensory deficit. The spinal epidural hematoma is one of the potentially reversible pressure lesions on the spinal cord and roots, thus its diagnosis and treatment have a vital importance. Presently magnetic resonance imaging (MRI) is considered as the first diagnostic method of choice, which can show a biconvex hematoma in the epidural space with welldefined borders that taper towards up and down. In case of lacking MRI, computed tomography scan could be used .

The initial surgical intervention is a general treatment for SSEH . If neurologic deficit was complete, decompressive surgery should be performed within 36 hours . Conservative treatment has been suggested just in cases that neurologic deficit improves in the early phase of disease or coagulopathy disorder subjects . Functional recovery mostly depends on duration of symptoms at presentation and its improving after 72 hours is rare, although some cases improved without surgery has been reported. Two cases were showed by Hentsched et al. in 2001, one with complete quadriplegia and another one with complete paraplegia that both of them were cured without surgery and had good neurological function during three months following. Another study was performed by Kim T and colleagues that 15 patients were evaluated in to two groups. Ten patients underwent decompressive surgery and the rest conservative treatment. These two groups did not show any difference in initial neurological status after treatment. It seems that the hematoma length is a clue in aspect of spontaneous recovery happens in some SSEH cases. In confronting with a patient complains from the pain, one of the important and priority duties of the physician is relieving the pain. But it is not enough alone and a logical diagnosis is required for justify the pain. Of course noticing to the limited time and facilities in the ED, sometimes there is no possibility to the definitive diagnosis. However, at least it should be rule out the critical causes and only in such condition, it is reasonable to discharge the patient to outpatient follow-up.

In the case of unknown psychiatric and neurologic manifestations, measuring serum level of thyroid hormones and CSF titer of anti-thyroid antibodies could be helpful in limitation of differential diagnosis and timely initiation of proper treatment.

\section{Refrences}

[1]. Deyo RA, Mirza SK, Martin BI. Back pain prevalence and visit rates: estimates from US national surveys, 2002. Spine. 2006;31(23):2724-7.

[2]. Ghafarzad A, Tagizadieh A, Moharamzadeh P, Majidi G. Thoracic Pneumorrhachis in Patient with Lumbar Fractures; a Case Report. Emergency. 2014;2(2):96-7.

[3]. Hosseini M, Karami Z, Janzadenh A, et al. The Effect of Intrathecal Administration of Muscimol on Modulation of Neuropathic Pain Symptoms Resulting from Spinal Cord Injury; a Experimental Study. Emergency.2014;2(4):11-8.

[4]. Binder DK, Sonne DC, Lawton MT. Spinal epidural hematoma. Neurosurg Q. 2004;14(1):51-9.

[5]. Miyagi Y, Miyazono M, Kamikaseda K. Spinal epidural vascular malformation presenting in association with a spontaneously resolved acute epidural hematoma: Case report. J Neurosurg. 1998;88(5):909-11.

[6]. Adam M, Leblebici B, Akman MN. Spontaneous spinal epidural hematoma related to warfarin therapy: A case report. J Back Musculoskelet Rehabil. 2007;20(1):11-4.

[7]. Marx JA, Hockberger RS, Walls RM, Adams GA. Rosen's emergency medicine: concepts and clinical practice. 6th ed. Philadelphia: Mosby Incorporated; 2010. pp. 605-675.

[8]. Matsumura A, Namikawa T, Hashimoto R, et al. Clinical management for spontaneous spinal epidural hematoma: diagnosis and treatment. Spine J. 2008;8(3):534-7.

[9]. Riaz S, Jiang H, Fox R, Lavoie M, Mahood JK. Spontaneous spinal epidural hematoma causing Brown-Sequard syndrome: case report and review of the literature. J Emerg Med. 2007;33(3):241-4.

[10]. Liu Z, Jiao Q, Xu J, Wang X, Li S, You C. Spontaneous spinal epidural hematoma: analysis of 23 cases. Surg Neurol. 2008;69(3):253-60.

[11]. Tailor J, Dunn IF, Smith E. Conservative treatment of spontaneous spinal epidural hematoma associated with oral anticoagulant therapy in a child. Childs Nerv Syst. 2006;22(12):1643-5.

[12]. Groen R. Non-operative treatment of spontaneous spinal epidural hematomas: a review of the literature and a comparison with operative cases. Acta Neurochir (Wien) 2004;146(2):103-10. 